\title{
Avaliação da influência do estresse e ansiedade nas disfunções temporomandibulares
}

\author{
Assessment of the influence of stress and anxiety on temporomandibular disorders \\ Evaluación de la influencia del estrés y la ansiedad en los trastornos temporomandibulares
}

Recebido: 2409/2021 | Revisado: 05/10/2021 | Aceito: 09/10/2021 | Publicado: 11/10/2021

\author{
Lorena Amaral Moreira \\ ORCID: https://orcid.org/0000-0002-0612-5141 \\ Universidade de Fortaleza, Brasil \\ E-mail: lorena.moreira98@gmail.com \\ Aretha de Magalhães e Souza \\ ORCID: https://orcid.org/0000-0002-0119-9360 \\ Universidade de Fortaleza, Brasil \\ E-mail: arethamgs@gmail.com \\ Maíra de Oliveira Viana Rela \\ ORCID: https://orcid.org/0000-0001-9057-948X \\ Universidade de Fortaleza, Brasil \\ E-mail: mairaoviana@unifor.br \\ João Esmeraldo Frota Mendonça \\ ORCID: https://orcid.org/0000-0001-6895-4597 \\ Universidade de Fortaleza, Brasil \\ E-mail: jesmeraldo@unifor.br
}

\begin{abstract}
Resumo
Objetivo: Analisar a influência do estresse e da ansiedade nos graus de severidade da disfunção temporomandibular (DTM). Metodologia: tratou-se de um estudo descritivo com abordagem quantitativa, realizado através de plataformas digitais no período de abril a junho de 2021. Foram inclusos no estudo indivíduos com a disfunção temporomandibular. E foram exclusos aqueles diagnosticados com fibromialgia, distúrbios neurológicos centrais ou periféricos e transtorno depressivo. Previamente foi realizada o compartilhamento do link do Google Forms® para indivíduos com queixa de quadro clínico de DTM, seguido ao preenchimento do formulário por parte dos participantes, que teve duração entre 10 e 15 minutos. O formulário consta de 4 seções: Identificação, Índice anamnésico de Fonseca, Inventário de Ansiedade Traço-Estado (IDATE) e Escala de Estresse Percebido (PSS-14). Resultados: 54 indivíduos participaram do estudo, sendo $88,9 \%$ do sexo feminino. Na classificação de Fonseca apresentou prevalência do grau de severidade leve (51,9\%). Quando aos dados do IDATE-E, 32 participantes apresentaram "baixo nível de ansiedade" (59,3\%) e no IDATE-T, 31 participantes "médio nível de ansiedade" (57,4\%), apresentando a menor contagem de 5 para "alto nível de ansiedade" $(9,3 \%)$. Na PSS-14, foram encontrados escores que variaram de 12 a 49 . A média do escore foi de $29,06( \pm 8,5)$, e 50 participantes apresentaram "médio nível de percepção" de estresse (92,6\%). Conclusão: Observou-se correlação significativa entre a ansiedade-traço e a DTM $(p<0,05)$.
\end{abstract}

Palavras-chave: Síndrome da Articulação Temporomandibular; Ansiedade; Estresse psicológico.

\begin{abstract}
Objective: To analyze the influence of stress and anxiety on the severity degrees of temporomandibular disorders (TMD). Methodology: this was a descriptive study with a quantitative approach, carried out through digital platforms from April to June 2021. Individuals with temporomandibular disorder were included in the study. And those diagnosed with fibromyalgia, central or peripheral neurological disorders and depressive disorder were excluded. Previously, the Google Forms $®$ link was shared for individuals complaining of a clinical TMD condition, followed by the filling out of the form by the participants, which lasted between 10 and 15 minutes. The form consists of 4 sections: Identification, Fonseca Anamnestic Index, State-Trait Anxiety Inventory (STAI) and Perceived Stress Scale (PSS-14). Results: 54 individuals participated in the study, being $88.9 \%$ female. The Fonseca classification showed a prevalence of mild severity (51.9\%). Regarding the IDATE-E data, 32 participants had "low level of anxiety" (59.3\%) and in the IDATET, 31 participants had "medium level of anxiety" (57.4\%), with the lowest score of 5 for "high level of anxiety" (9.3\%). On the PSS-14, scores ranging from 12 to 49 were found. The mean score was $29.06( \pm 8.5)$, and 50 participants had an "average level of perception" of stress $(92.6 \%)$. Conclusion: There was a significant correlation between trait anxiety and TMD $(\mathrm{p}<0.05)$.
\end{abstract}

Keywords: Temporomandibular Joint Syndrome; Anxiety; Stress, psychological.

\section{Resumen}

Objetivo: Analizar la influencia del estrés y la ansiedad en los grados de severidad de los trastornos temporomandibulares (TTM). Metodología: se trata de un estudio descriptivo con enfoque cuantitativo, realizado a 
través de plataformas digitales de abril a junio de 2021. Se incluyeron en el estudio individuos con trastorno temporomandibular. Y se excluyó a los diagnosticados de fibromialgia, trastornos neurológicos centrales o periféricos y trastorno depresivo. Anteriormente, el enlace de Google Forms ${ }^{\circledR}$ se compartía para las personas que se quejaban de una afección clínica de TMD, seguido del llenado del formulario por parte de los participantes, que duró entre 10 y 15 minutos. El formulario consta de 4 secciones: Identificación, Índice Anamnésico de Fonseca, Inventario de Ansiedad Rasgo-Estado (STAI) y Escala de Estrés Percibido (PSS-14). Resultados: participaron en el estudio 54 individuos, siendo el 88,9\% mujeres. La clasificación de Fonseca mostró una prevalencia de gravedad leve $(51,9 \%)$. Con respecto a los datos del IDATE-E, 32 participantes tenían "bajo nivel de ansiedad" (59,3\%) y en el IDATE-T, 31 participantes tenían "nivel medio de ansiedad" (57,4\%), con la puntuación más baja de 5 para nivel de ansiedad" (9,3\%). En el PSS14 se encontraron puntuaciones que iban de 12 a 49. La puntuación media fue de $29,06( \pm 8,5)$ y 50 participantes tenían un "nivel medio de percepción" de estrés $(92,6 \%)$. Conclusión: Hubo una correlación significativa entre el rasgo de ansiedad y TTM (p <0.05).

Palabras clave: Síndrome de la Articulación Temporomandibular; Ansiedad; Estrés psicológico.

\section{Introdução}

Determinada como um termo coletivo que engloba muitas alterações clínicas que abalam os músculos mastigatórios, articulação temporomandibular e elementos associados, ou ambos, a disfunção temporomandibular (DTM) é vista como uma subclassificação das disfunções musculoesqueléticas e tipicamente apresenta um curso recorrente ou crônico (Vasconcelos et al., 2019).

A DTM manifesta etiologia complexa, relacionada a condições predisponentes, iniciadores e perpetuantes, como alterações oclusais, hábitos parafuncionais, estresse, ansiedade, ou anormalidades no disco intra-articular. Assim, tais fatores podem estar relacionados à ocorrência de inflamações articulares, danos e dores musculares, ou espasmos. Desta forma, entre os sinais e sintomas mais comuns estão ruídos articulares, cefaleias, dores na região pré-auricular, otalgias, dores na face e na cervical, cansaço muscular, desvio da trajetória da mandíbula durante o movimento, limitação na abertura de boca, além de sensibilidade dentária, causando grande desconforto e prejuízo da qualidade de vida (Sassi, Silva, Santos, \& Andrade, 2018).

Posto isso, a DTM é uma síndrome a qual pode manifestar apenas pequenos incômodos nos pacientes como também dolorosos sintomas, que por muitas vezes podem ser impossibilitantes. Entre os agentes causadores e de alerta para a DTM temos o bruxismo em vigília ou noturno, alteração oclusal, estresse e dentre outros fatores psicossociais. Ocasiona impactos negativos no bem-estar, saúde, qualidade de vida e trabalho em decorrências da dor causada pela disfunção, sendo vista com mais frequência no gênero feminino. A dor ocasionada pela DTM pode-se tornar crônica devido os aspectos psicossociais (Urbani, Jesus \& Cozendey-Silva, 2019).

Neste sentido, em estudos realizados com estudantes universitários foi visto uma alta propensão ao desenvolvimento de quadros de ansiedade e depressão, esses, por sua vez, influenciarão o aparecimento de sintomas associados à DTM. Apresenta com mais frequência uma faixa etária entre 20 e 40 anos e em relação ao sexo, a prevalência entre as mulheres chega a ser de três a nove vezes maior quando comparada com homens (Soares, Coelho, Moreno, Almeida, \& Haddad, 2020).

Segundo Costa et al. (2020), a disfunção influência em diversos aspectos da vida do indivíduo que a possui, trazendo vários acometimentos para sua saúde, sendo uma delas a dor. Os resultados desse estudo mostram que em atividades como o sono, 14 pessoas $(52 \%)$ referiram muita dificuldade para dormir em virtude da dor e $37 \%$ relataram a perda frequente do apetite devido a dor. Quanto à qualidade de vida de pessoas diagnosticadas com DTM, o prejuízo em aspectos como a dor persistente (74\%), sensação de desconforto ao comer (48\%) e tensão pelo problema bucal (37\%). Demonstrando que a disfunção é capaz de interferir na qualidade de vida das pessoas.

Desta forma, o estado de ansiedade é conceituado como um estado emocional transitório ou condição do organismo humano que é caracterizada por sentimentos desagradáveis de tensão e apreensão, dessa forma o organismo reage ao estímulo que é interpretado pelo indivíduo como ameaça, causando um desequilíbrio emocional e físico (Carmo \& Labes Ferreira, 2016; Sousa, Moreira, \& Santos, 2016). A ansiedade desencadeia hábitos parafuncionais e tensão muscular, levando ao aparecimento 
dos sinais e sintomas de DTM e o sofrimento psicológico significativo, levando aos distúrbios de humor, com elevados índices de estresse e ansiedade (Costa, Freitas, \& Barbosa, 2020).

O estresse, somado a outros aspectos, como uma má oclusão pode ser o estopim para o surgimento de uma DTM. Certas patologias da mucosa bucal, podem ser desencadeadas por determinado grau de estresse, levando o paciente à patologias ou agravando doenças já existentes. A própria DTM é um fator causal de estresse, pois seus sinais e sintomas quando presentes por um tempo prolongado, podem acarretar desordens psicológicas como estresse e até mesmo depressão em um nível mais intenso (Graciola \& Silveira, 2013).

Pessoas que sofrem com a disfunção temporomandibular se consideram muitos estressadas (Graciola \& Silveira, 2013). Além disso, compondo a natureza fisiológica dos seres humanos, o estresse está associado à capacidade de adaptação diante de um acontecimento ou circunstância importante. Todavia, no momento que o estresse excede as ações físicas, emocionais e cognitivas do homem em enfrentar situações estressoras ele torna-se excessivo ou resistente, gerando impacto negativo no organismo, podendo ocasionar um quadro clínico mórbido (Urbani, Jesus \& Cozendey-Silva, 2019).

Portanto, existe uma associação do estresse com a DTM, percebido pela forte ligação da síndrome com condições estressoras como depressão, transtorno do sono, alterações psicológicas e ansiedade, ocorrendo não exclusivamente no surgimento da disfunção, como também no seu desenvolvimento e tratamento. Nesse contexto, é fundamental avaliar a ação dos aspectos psíquicos nesses pacientes (Urbani, Jesus \& Cozendey-Silva, 2019).

Levando em consideração que o estresse e a ansiedade têm intima conexão com a DTM, o presente estudo objetivou analisar a influência do estresse e da ansiedade nos graus de severidade da disfunção temporomandibular.

\section{Metodologia}

Foi realizado um estudo descritivo com abordagem quantitativa, através de plataformas digitais, objetivando analisar a influência do estresse e da ansiedade nos graus de severidade da disfunção temporomandibular. Este estudo respeitou os preceitos éticos da Resolução 466/12 do CNS/MS que rege os princípios éticos da pesquisa envolvendo seres humanos sendo aprovado pelo comitê de ética com o parecer número 3.711.323.

A amostra foi constituída por 62 participantes com queixa de quadro clínico de DTM, que receberam ou viram a mensagem da realização da pesquisa nas mídias sociais, como Instagram® e WhatsApp®, entre o período de abril a junho de 2021.

Foram incluídos no estudo participantes com idade entre 18 e 70 anos, de ambos os gêneros e com grau de severidade da disfunção temporomandibular segundo o índice de Fonseca. Foram exclusos aqueles participantes diagnosticados com fibromialgia, distúrbios neurológicos centrais ou periféricos e transtorno depressivo.

O estudo foi dividido em dois momentos. O primeiro momento reservado para a divulgação da realização da pesquisa e o compartilhamento do link do Google Forms $®$ através das mídias sociais pela pesquisadora, e o segundo momento destinado ao preenchimento do formulário por parte dos participantes, que teve duração entre 10 e 15 minutos.

O formulário consta de 4 seções: Identificação, Índice anamnésico de Fonseca, Inventário de Ansiedade Traço-Estado (IDATE) e Escala de Estresse Percebido (PSS-14).

Inicialmente todos os participantes responderam sobre sua identificação e foram avaliados para classificação dos graus de severidade da disfunção temporomandibular segundo o Índice Anamnésico de Fonseca.

Para a classificação dos graus de severidade segundo o Índice Anamnésico de Fonseca, os voluntários responderam aos 10 itens compostos no mesmo, contendo 3 tipos de resposta: Sim, Às vezes ou Não. A resposta 'Sim' equivale a 10 pontos, a

‘Às vezes' são 5 pontos e o ‘Não' 0 pontos. Com a somatória dos pontos atribuídos obtém-se um índice anamnésico que permite 
classificar os participantes em categorias de severidade de sintomas: sem DTM - 0 a 15 pontos, DTM leve - 20 a 40 pontos, DTM moderada - 45 a 65 e DTM severa - 70 a 100 pontos (Chaves, Oliveira, \& Grossi, 2008).

Na seção seguida, foi aplicado um questionário de auto-avaliação, o Inventário de Ansiedade Traço-Estado - IDATE, responsável por avaliar a presença de ansiedade. Este inventário é composto por 40 afirmações e dividido igualmente em duas partes.

$\mathrm{Na}$ Parte I, os voluntários serão instruídos a indicar a intensidade de seus sentimentos de ansiedade em um determinado momento (ansiedade do estado), ou seja, como se sente "agora, neste momento" em relação as 20 afirmações, usando pontuações que variam de 1 (absolutamente não) a 4 (muitíssimo) (Santos \& Galdeano, 2009).

Na Parte II, os voluntários descreverão como eles geralmente se sentem (traço de ansiedade) relatando a frequência de seus sintomas de ansiedade, em relação as outras 20 afirmações, novamente usando pontuações variando de 1 (quase nunca) a 4 (quase sempre) (Santos \& Galdeano, 2009).

A pontuação total de cada parte pode variar entre 20 e 80, com escores mais altos indicando níveis mais altos de ansiedade, e corresponde ao nível de ansiedade, sendo que de 20 a 40 pontos equivalem a baixo nível de ansiedade; 41 a 60 pontos, a médio nível de ansiedade; e 60 a 80 pontos, a alto nível de ansiedade (Santos \& Galdeano, 2009).

Posteriormente, na última seção do formulário foi aplicado a Escala de Estresse Percebido (PSS-14), sendo utilizada para avaliar o grau no qual os indivíduos percebem as situações como estressantes.

A versão do PSS utilizada possui 14 questões com opções de resposta que variam de zero a quatro ( $0=$ nunca; $1=$ quase nunca; $2=$ às vezes; $3=$ quase sempre; $4=$ sempre). As questões com conotação positiva $(4,5,6,7,9,10$ e 13$)$ têm sua pontuação somada invertida da seguinte maneira: $0=4,1=3,2=2,3=1$ e $4=0$. As demais questões são negativas e devem ser somadas diretamente (Preto et al., 2020; Dias, Silva, Maroco, \& Campos, 2015).

O escore total é calculado pela soma das pontuações destas 14 questões e pode variar de zero a 56. Assim, quanto maior o escore na escala, maior o nível de percepção de estresse. Valores acima do percentil 75 (42 pontos) devem ser considerados indicativos de "alto nível de percepção" de estresse, percentis entre 25 e 75 foram considerados como "médio nível de percepção" e o percentil abaixo de 25 foi considerado como "baixo nível de percepção" (Preto et al., 2020; Dias, Silva, Maroco, \& Campos, 2015).

Os dados foram coletados e analisados através da estatística descritiva, com o auxílio do software estatístico SPSS (Statistical Package For Social Sciencies), versão 28 e para a comparação dos dados foi realizado o teste Qui-quadrado de Pearson.

\section{Resultados}

O presente estudo contou com a participação de 62 indivíduos, no qual 8 foram retirados pelo critério de exclusão, totalizando a amostra com 54 participantes. Tendo como maior predomínio o sexo feminino (88,9\%), com faixa etária entre 18 e 64 anos, sendo a idade média de 29,31 anos $( \pm 12,13)$. Quanto a ocupação, encontrou-se que a maioria é estudante $(51,9 \%)$ (Tabela 1). 
Tabela 1 - Características descritivas dos participantes.

\begin{tabular}{|c|c|c|c|c|c|c|c|}
\hline Variáveis & & $\mathbf{n}$ & $\%$ & Média & Mín/Máx & Moda & $\begin{array}{l}\text { Desvio } \\
\text { Padrão }\end{array}$ \\
\hline \multirow{2}{*}{ Sexo } & Feminino & 48 & $88,9 \%$ & & & & \multirow{2}{*}{0,317} \\
\hline & Masculino & 6 & $11,1 \%$ & 1,11 & & & \\
\hline Idade & & 54 & & 29,31 & $18 / 64$ & 21 & 12,13 \\
\hline \multirow{2}{*}{ Profissão } & Estudante & 28 & $51,9 \%$ & 1,48 & & & \multirow{2}{*}{0,504} \\
\hline & Não estudante & 26 & $48,1 \%$ & & & & \\
\hline
\end{tabular}

Legenda: $\mathrm{n}=$ Número de participantes; \%=percentual. Fontes: Autores.

No que se refere ao grau de acometimento da disfunção temporomandibular, segundo o Índice Anamnésico de Fonseca, 28 dos participantes apresentaram "DTM leve" (51,9\%), 14 "DTM moderada" (25,9\%) e 12 "DTM severa" (22,2\%), com média de 47,59 ( $\pm 21,64)$.

Quanto as médias encontradas no IDATE-E e no IDATE-T apresentaram um nível de "Ansiedade Moderado" na amostra estudada (Tabela 2), sendo que no IDATE-E 16 participantes apresentaram "baixo nível de ansiedade" (29,6\%), 32 "médio nível de ansiedade" (59,3\%) e 6 participantes "alto nível de ansiedade" (11,1\%). No IDATE-T, 18 participantes apresentaram "baixo nível de ansiedade" (33,3\%), 31 "médio nível de ansiedade" (57,4\%) e 5 "alto nível de ansiedade" (9,3\%).

$\mathrm{Na}$ aplicação da Escala de Estresse Percebido (PSS-14), foram encontrados escores que variaram de 12 a 49. A média do escore foi de 29,06 $( \pm 8,5)$. Foram vistas, ainda, médias mínima e máxima de 1,43 e 3,11, respectivamente, dentre as 14 questões, conforme dados da Tabela 3. Apenas 3 participantes ultrapassaram o percentil 75, sendo considerados com "alto nível de percepção" de estresse (5,6\%). Em contrapartida, 50 participantes apresentaram "médio nível de percepção" de estresse (92,6\%) e 1 "baixo nível de percepção" de estresse (1,9\%).

Relacionando os graus de DTM com os níveis de ansiedade, verificou-se que só houve associação significante entre os graus de DTM e a ansiedade-traço ( $\mathrm{p}<0,05)$, de modo que o nível médio foi mais frequente nos sujeitos com DTM leve, como mostra a Tabela 4.

Tabela 2 - Descritivos dos valores do Inventário de Ansiedade Estado-Traço.

\begin{tabular}{ccccc}
\hline & Médias & Desvio Padrão & Valor Mínimo & Valor Máximo \\
\hline IDATE-E & 46,67 & $\pm 11,93$ & 26 & 78 \\
\hline IDATE-T & 46,31 & $\pm 11,49$ & 27 & 78 \\
\hline
\end{tabular}

Fontes: Autores.

\section{Discussão}

Várias pesquisas são coordenadas para verificar a DTM em diversos públicos com distintas atividades e intervalo de idade (Reis et al., 2021). É de suma importância identificar os sinais e sintomas da DTM para realizar o diagnóstico antecipado dessa disfunção (Sousa, Moreira, \& Santos, 2016).

Tem sido reconhecida a atuação da ansiedade e do estresse sobre o limiar de dor à pressão nos músculos mastigatórios e quanto aos relatos subjetivos da dor. Nesse contexto, a utilização de questionários e índices tem sido amplamente difundido na literatura, em especial quando validados, de fácil interpretação e aplicação, com objetivo de uniformizar a comparação dos dados (Oliveira, 2017). 
Tabela 3 - Dados da PSS, em ordem decrescente de participantes.

\begin{tabular}{|c|c|c|c|c|c|}
\hline $\mathbf{N}^{\mathbf{O}}$ & $\begin{array}{l}\text { No último mês com que frequência você } \\
\text { tem... }\end{array}$ & Mín/Máx & Média & Moda & Desvio Padrão \\
\hline 12 & $\begin{array}{l}\text { Você tem se encontrado pensando sobre as } \\
\text { coisas que deve fazer? }\end{array}$ & $1 / 4$ & 3,11 & 4 & 0,945 \\
\hline 3 & Você tem se sentido nervoso e "estressado"? & $0 / 4$ & 2,48 & 2 & 0,966 \\
\hline $\mathbf{1}$ & $\begin{array}{l}\text { Você tem ficado triste por causa de algo que } \\
\text { aconteceu inesperadamente? }\end{array}$ & $0 / 4$ & 2,46 & 2 & 1,004 \\
\hline 8 & $\begin{array}{l}\text { Você tem achado que não conseguiria lidar } \\
\text { com todas as coisas que você tem que fazer? }\end{array}$ & $0 / 4$ & 2,39 & 2 & 0,979 \\
\hline 13 & $\begin{array}{l}\text { Você tem conseguido controlar a maneira } \\
\text { como gasta seu tempo? }\end{array}$ & $0 / 4$ & 2,22 & 2 & 1,003 \\
\hline 7 & $\begin{array}{l}\text { Você tem sentido que as coisas estão } \\
\text { acontecendo de acordo com a sua vontade? }\end{array}$ & $0 / 4$ & 2,15 & 2 & 1,106 \\
\hline 2 & $\begin{array}{l}\text { Você tem se sentido incapaz de controlar as } \\
\text { coisas importantes em sua vida? }\end{array}$ & $0 / 4$ & 2,11 & 2 & 1,127 \\
\hline 11 & $\begin{array}{l}\text { Você tem ficado irritado porque as coisas que } \\
\text { acontecem estão fora do seu controle? }\end{array}$ & $0 / 4$ & 2,09 & 2 & 1,186 \\
\hline 10 & $\begin{array}{l}\text { Você tem sentido que as coisas estão sob o } \\
\text { seu controle? }\end{array}$ & $0 / 4$ & 2,04 & 2 & 1,009 \\
\hline 14 & $\begin{array}{l}\text { Você tem sentido que as dificuldades se } \\
\text { acumulam a ponto de você acreditar que não } \\
\text { pode superá-las? }\end{array}$ & $0 / 4$ & 1,80 & 2 & 1,188 \\
\hline 4 & $\begin{array}{l}\text { Você tem tratado com sucesso dos problemas } \\
\text { difíceis da vida? }\end{array}$ & $0 / 4$ & 1,65 & 2 & 0,781 \\
\hline 9 & $\begin{array}{l}\text { Você tem conseguido controlar as irritações } \\
\text { em sua vida? }\end{array}$ & $0 / 3$ & 1,61 & 2 & 0,856 \\
\hline 5 & $\begin{array}{l}\text { Você tem sentido que está lidando bem as } \\
\text { mudanças importantes que estão ocorrendo } \\
\text { em sua vida? }\end{array}$ & $0 / 4$ & 1,52 & $2 *$ & 0,885 \\
\hline 6 & $\begin{array}{l}\text { Você tem se sentido confiante na sua } \\
\text { habilidade de resolver problemas pessoais? }\end{array}$ & $0 / 3$ & 1,43 & 1 & 0,924 \\
\hline
\end{tabular}

Legenda: PSS= Perceived Stress Scale; *= Há vários modos. O menor valor é mostrado. Fontes: Autores.

Na análise do predomínio de DTM, o gênero é visto como uma significativa variável, corroborando com vários estudos nos quais sugerem a propensão de sintomas relacionados à DTM em jovens adultas do sexo feminino (Moreno et al., 2021), atestando assim os achados dessa pesquisa em relação ao predomínio da disfunção temporomandibular em mulheres.

Neste sentido, a maior prevalência deste gênero deve estar associada ao fato de as mulheres procurarem tratamento com maior frequência, ou seja, são mais cuidadosas e atenciosas em relação a saúde comparadas aos homens (Pinto, Leite, Sampaio, \& Sanchez, 2017). A associação do gênero feminino e da DTM pode ser justificada também pelas condições fisiológicas características das mulheres, na qual as tornam mais sensíveis em momentos de tensão física e psíquica, além de dificultar a estabilidade da ATM (Costa, Freitas, \& Barbosa, 2020).

No que se refere ao grau de severidade da DTM, a maior prevalência foi "DTM leve" (51,9\%). Os dados apresentam divergência aos expostos na literatura, em que estudos direcionados à análise da severidade expõem prevalência para o grau “moderado" (Reis et al., 2021; Sousa, Moreira, \& Santos, 2016). Todavia, nos dois estudos se observa que o gênero feminino foi o mais afetado. 
Tabela 4 - Avaliação dos graus de disfunção temporomandibular segundo o nível de ansiedade.

\begin{tabular}{|c|c|c|c|c|c|c|c|c|c|c|}
\hline & \multirow{3}{*}{ Variáveis } & \multicolumn{6}{|c|}{ DTM } & \multirow{3}{*}{\multicolumn{2}{|c|}{ Total }} & \multirow{3}{*}{$\begin{array}{l}\text { Valor } \\
\text { do p }\end{array}$} \\
\hline & & \multicolumn{2}{|c|}{ Leve } & \multicolumn{2}{|c|}{ Moderada } & \multicolumn{2}{|c|}{ Severa } & & & \\
\hline & & $\mathrm{n}$ & $\%$ & $\mathrm{n}$ & $\%$ & $\mathrm{n}$ & $\%$ & & & \\
\hline \multirow{3}{*}{$\begin{array}{c}\text { Ansiedade } \\
\text { Estado }\end{array}$} & Baixo & 11 & 68,8 & 2 & 12,5 & 3 & 18,8 & 16 & 100,0 & \multirow{3}{*}{$\mathrm{p}=0,100$} \\
\hline & Médio & 16 & 50,0 & 9 & 28,1 & 7 & 21,9 & 32 & 100,0 & \\
\hline & Alto & 1 & 16,7 & 3 & 50,0 & 2 & 33,3 & 6 & 100,0 & \\
\hline \multirow{4}{*}{$\begin{array}{c}\text { Ansiedade } \\
\text { Traço }\end{array}$} & Baixo & 13 & 72,2 & 3 & 16,7 & 2 & 11,1 & 18 & 100,0 & \multirow{3}{*}{$\mathrm{p}=0,024^{*}$} \\
\hline & Médio & 14 & 45,2 & 9 & 29,0 & 8 & 25,8 & 31 & 100,0 & \\
\hline & Alto & 1 & 20,0 & 2 & 40,0 & 2 & 40,0 & 5 & 100,0 & \\
\hline & Grupo total & 28 & 51,9 & 14 & 25,9 & 12 & 22,2 & 54 & 100,0 & \\
\hline
\end{tabular}

Legenda: $\mathrm{n}=$ Número de participantes; $\%=$ percentual; *= A correlação é significativa no nível 0,05 (2 extremidades). Qui-quadrado de Pearson. Fontes: Autores.

Em relação à relação entre o grau de severidade da DTM e a profissão, tivemos um número elevado de participantes estudante (51,9\%). A prevalência foi no grupo com DTM leve, contendo 16 estudantes (57,1\%). Nos resultados do estudo de Pinto et al. (2017), percebeu-se que foi considerável a parcela de universitários com sinais e sintomas de DTM (66,3\%), o que também confere em resultados encontrados no estudo de Bezerra et al. (2012), que encontraram prevalência de DTM, nos seus diferentes graus, em 62,5\% dos participantes universitários.

Assim, devido ser um dos principais estimuladores de tensão muscular, o estado emocional ocasiona a progressão das desordens musculoesqueléticas. Diversos estudos relacionam a depressão e a ansiedade com o desenvolvimento da síndrome da articulação temporomandibular (Bezerra et al., 2012; Reis et al., 2021; Sousa, Moreira, \& Santos, 2016).

No estudo sobre a ansiedade são apontados dois princípios diferentes: A ansiedade- estado (IDATE-E), na qual está relacionada a reações emocionais desagradáveis, singularizado por sentimentos de apreensão e preocupação, decorrentes de um estado momentâneo e passageiro, e a ansiedade-traço (IDATE-T), a qual é caracterizada pelas diferenças individuais, com tendência para identificar situações estressantes como ameaçadoras e perigosas, e reagir de maneira peculiar a sua personalidade (Cordeiro \& Freire, 2016).

A relação do nível de ansiedade para cada grupo de DTM, a maior média de ansiedade na sua modalidade Estado foi identificado nos indivíduos do grupo com DTM moderada $(54,00)$ do sexo feminino. Em referência ao índice de ansiedade na sua modalidade Traço, a maior média manifestou-se no grupo feminino de DTM moderada $(53,00)$ e a menor média manifestouse no grupo masculino com DTM leve $(34,00)$. Em ambas as modalidades prevaleceu maiores números para o sexo feminino, achado este que entra em concerto com outras pesquisas contendo o inventário (IDATE) (Reis et al., 2021; Sousa, Moreira, \& Santos, 2016).

Nos resultados do estudo de Reis et al. (2021), mostraram a existência para todos os níveis de ansiedade, entende-se que a predominância do estado ansioso para todos os níveis seja pelas exigências impostas aos alunos, análise esta que se aparenta ao encontrado no estudo de Sousa et al. (2016), que mostra prevalência da ansiedade em grau moderado em mais de $92 \%$ dos universitários estudados, sendo estes diagnosticados com DTM.

Já Lima et al. (2017), aplicaram apenas o Inventário de Ansiedade Traço (IDATE T) em alunos concludentes, mostrando a existência de ansiedade em níveis altos para 52,9\%. Esse estudo corrobora com os supracitados, pois mostrou a presença de ansiedade-estado e ansiedade-traço em níveis moderados para 59,3\% e 57,4\%, respectivamente. 
Correlacionando o nível de percepção de estresse com o grau de severidade da DTM, 50 dos indivíduos estudados (92,6\%) apresentaram médio nível de percepção de estresse, em todos os graus de severidade. Já a menor frequência manifestou-se no baixo nível de percepção de estresse (1,9\%).

O estresse causado pela lembrança constante das tarefas a serem realizadas, sensação de nervosismo e de estressado, para lidar com o estresse percebido, item 12 e 3, respectivamente, foram os fatores de maior pontuação na PSS (Tabela 3). Corroborando com estudos de Yosetake et al. (2018), a qual na aplicação da Escala de Estresse Percebido, foram encontrados escores que variaram de 14 a 45, média de escore de 29,3, e os fatores de maior pontuação também foram as lembranças constantes das tarefas e sensação de nervoso e estressado.

Exercendo impacto tanto na progressão da DTM quanto na exacerbação dos seus sinais, o estresse por si próprio não é bastante para ocasionar problemas na saúde dos indivíduos. O início da síndrome necessita da agregação com outras elementos, por exemplo, a competência emocional particular para enfrentar os agentes estressores, ou a acumulação de fontes estressoras ou, como também, a propensão para o desenvolvimento de certas patologias (Pinto et al., 2021; Urbani, Jesus, \& CozendeySilva, 2019).

Este estudo sofreu limitações quanto ao número de participantes e a abordagem da coleta de dados devido a pandemia de COVID-19, dificultando a investigação mais detalhada e específica sobre o assunto.

\section{Conclusão}

Todos os participantes apresentam diagnóstico sintomatológico para a síndrome da articulação temporomandibular, com predomínio do sexo feminino e grau de severidade leve. Os achados apontam que a existência da disfunção esteja ligada com a exigências severas, ações de hábitos parafuncionais, eventos estressantes e momento de ansiedade relatado pelos participantes.

Por fim, de acordo com os dados apresentados não tivemos relação significativa entre o estresse e os graus de severidade da DTM, e nem do ansiedade-estado com a DTM, porém tivemos correlação significativa entre a ansiedade-traço e a DTM.

Visa-se novos estudos que abordem a relação da ansiedade e do estresse com DTM e também a realização de estudos que abordem uma intervenção precoce, pois observou-se um predomínio de estudantes neste estudo.

\section{Agradecimentos}

Sinceros agradecimentos à Equipe Multidisciplinar em Disfunção e Dor Orofacial (EMDOF) da Universidade de Fortaleza, em especial a Fundação Cearense de Apoio ao Desenvolvimento Científico e Tecnológico (FUNCAP) por proporcionar e contribuir com a realização dessa pesquisa.

\section{Referências}

Bezerra, B. P. N., Ribeiro, A. I. A. M., Farias, A. B. L. D., Farias, A. B. L. D., Fontes, L. D. B. C., Nascimento, S. R. D., ... \& Adriano, M. S. P. F. (2012). Prevalência da disfunção temporomandibular e de diferentes níveis de ansiedade em estudantes universitários. Revista Dor, 13, 235-242. 10.1590/S180600132012000300008 .

Carmo, L. S. do, \& Labes Ferreira, J. (2016). Stress into university students impacts the learning quality. Journal of Psychology \& Psychotherapy, 06(02), 1-4. 10.4172/2161-0487.1000248.

Chaves, T. C., Oliveira, A. S. de, \& Grossi, D. B. (2008). Principais instrumentos para avaliação da disfunção temporomandibular, parte I: Índices e questionários; uma contribuição para a prática clínica e de pesquisa. Fisioterapia e Pesquisa, 15(1), 92-100.

Cordeiro, R. A., \& Freire, V. (2016). Estado-Traço de ansiedade e vivências académicas em estudantes do $1^{\circ}$ ano do Instituto Politécnico de Portalegre. Millenium-Journal of Education, Technologies, and Health, (36).

Costa, F. A. S. da, Freitas, L. A. Q. de, \& Barbosa, R. de S. P. (2020). Doenças psicossociais nas disfunções temporomandibular e o impacto na qualidade de vida das mulheres. Revista Cathedral, 2(4), 31-38. 
Dias, J. C. R., Silva, W. R., Maroco, J., \& Campos, J. A. D. B. (2015). Escala de Estresse Percebido aplicada a estudantes universitárias: estudo de validação. Psychology, Community \& Health, 4(1), 1-13. 10.23668/psycharchives. 2277.

Graciola, J., \& Silveira, A. M. (2015). Avaliação da influência do estresse na prevalência de disfunções temporomandibulares em militares estaduais do Rio Grande do Sul. Journal of Oral Investigations, 2(1), 32-37. 10.18256/2238-510X/j.oralinvestigations.v2n1p32-37.

Lima, B. V. D. B. G., Trajano, F. M. P., Neto, G. C., Alves, R. S., Farias, J. A., \& Braga, J. E. F. (2017). Avaliação da ansiedade e autoestima em concluintes do curso de graduação em enfermagem. Revista de Enfermagem UFPE on line, 11(11), 4326-4333. 10.5205/1981-8963-v11i11a13440p4326-4333-2017.

Moreno, A. G. U. T., Bezerra, A. G. V., Alves-Silva, E. G., Melo, E. L. de, Martínez Gerbi, M. E. M. de, Bispo, M. E. A., ... \& de Menezes, M. R. A. (2021). Influência do estrógeno na modulação da dor na disfunção temporomandibular e sua prevalência no sexo feminino: revisão integrativa. Research, Society and Development, 10(2), e38510212453-e38510212453. 10.33448/rsd-v10i2.12453.

Oliveira, P. S. D. (2017). Relação entre estresse, ansiedade e disfunção temporomandibular. (Trabalho de Conclusão de Curso).

Pinto, A. C. M., Oliveira, A. M., Neto, D. A. S. da, Lins, F. H., Santos, J. P. dos, \& Sousa, M. N. A. de. (2021). Estresse psicológico e disfunções temporomandibulares: Revisão integrativa. The Open Brazilian Dentristy Journal, 1(1), 01-12.

Pinto, R. G. S., Leite, W. M. A., Sampaio, L. D. S., \& Sanchez, M. D. O. (2017). Associação entre sinais e sintomas de disfunção temporomandibular com depressão em universitários: estudo descritivo. Revista Dor, 18(3), 217-224. 10.5935/1806-0013.20170105.

Preto, V. A., Souza, A. L. T. de, Sousa, B. D. O. P., Fernandes, J. M., Souza Pereira, S. de, \& Cardoso, L. (2020). Associação entre fatores pessoais e ambientais com o estresse recente em estudantes de enfermagem. Research, Society and Development, 9(6), e113962572-e113962572. 10.33448/rsd-v9i6.2572.

Reis, K. S. dos, da Rocha, V. A., Neta, N. B. D., Cantinho, K. M. C. R., de Morais Gouveia, G. P., \& Carvalho, G. D. (2021). Prevalência e fatores associados à disfunção temporomandibular em estudantes de fisioterapia: estudo transversal. Research, Society and Development, 10(5), e37710514984-e37710514984. 10.33448/rsd-v10i5.14984.

Santos, M. D. L. D., \& Galdeano, L. E. (2009). Traço e estado de ansiedade de estudantes de enfermagem na realização de uma prova prática. Revista Mineira de Enfermagem, 13(1), 76-83

Sassi, F. C., Silva, A. P. da, Santos, R. K. S., \& Andrade, C. R. F. de. (2018). Tratamento para disfunções temporomandibulares: Uma revisão sistemática. Audiology - Communication Research, 23(0). 10.1590/2317-6431-2017-1871.

Soares, L. F. F., Coelho, L. M., Moreno, A., Almeida, D. A. de F., \& Haddad, M. F. (2020). Anxiety and depression associated with pain and discomfort of temporomandibular disorders. Brazilian Journal Of Pain, 3(2), 147-152. 10.5935/2595-0118.20200029.

Sousa, E. F. de, Moreira, T. R., \& Santos, L. H. G. (2016). Correlação do nível de ansiedade e da qualidade de vida com os sinais e sintomas da disfunção temporomandibular em universitários. Clínica e Pesquisa em Odontologia-UNITAU, 8(1), 16-21.

Urbani, G., Jesus, L. F. de, \& Cozendey-Silva, E. N. (2019). Síndrome da disfunção da articulação temporomandibular e o estresse presente no trabalho policial: Revisão integrativa. Ciência \& Saúde Coletiva, 24(5), 1753-1765. 10.1590/1413-81232018245.16162017.

Vasconcelos, R. S. N., Marques, L. A. R. V., Kuehner, M. C. P., Barroso, K. S. N., Dias, C. C., Carmo Filho, J. R. L., Fiallos, A. C. D. M., \& Fernandes, M. L. (2019). Fisioterapia na disfunção temporomandibular. Saúde (Santa Maria), 45(2), 13. 10.5902/2236583427266.

Yosetake, A. L., de Lima Camargo, I. M., Luchesi, L. B., da Silva Gherardi-Donato, E. C., \& Teixeira, C. A. B. (2018). Estresse percebido em graduandos de enfermagem. SMAD Revista Eletrônica Saúde Mental Álcool e Drogas (Edição em Português), 14(2), 117-124. 10.11606/issn.1806-6976.smad.2018.000336. 